\title{
Switchable phase diffusion in intrinsic Josephson junction arrays
}

J.C. Fenton ${ }^{\mathrm{a}^{*}}$, M. Korsah ${ }^{\mathrm{b}}$, C.R.M. Grovenor ${ }^{\mathrm{b}}$ and P.A. Warburton ${ }^{\mathrm{a}}$

${ }^{a}$ Department of Electronic and Electrical Engineering, University College London, Torrington Place, London WC1E

7JE, UK

${ }^{b}$ Department of Materials, University of Oxford, Parks Road, Oxford, OX1 3PH, UK

This article is available in journal format at http://dx.doi.org/10.1016/j.physc.2007.04.156

\section{Abstract}

We report current-voltage measurements on arrays of high-Tc intrinsic Josephson junctions showing a temperature dependence of the first critical current which is different from that of subsequent critical currents for an array with sub-micron dimensions. This can be explained by the change in the impedance seen by other array-junctions once one junction switches out of the supercurrent state, resulting in a switching-off of thermally activated phase-diffusion. (C) 2001 Elsevier Science. All rights reserved

Keywords: c-axis transport; Tl2Ba2CaCu2O8; environmental impedance

\footnotetext{
* Corresponding author. Tel.: +44-207-679-0640; e-mail: j.fenton@ucl.ac.uk.
} 
The c-axis transport properties of anisotropic cuprate superconductors strongly suggest that they form an array of Josephson junctions; these arrays are referred to as "intrinsic Josephson junction" (IJJ) arrays [1]. In an array of $\mathrm{N}$ identical IJJs, each junction can be in the supercurrent state or the voltage state. Therefore there exist $\mathrm{N}+1$ possible states for the array of junctions. These we label the " 0 " state (where all $N$ junctions are in the supercurrent state), the " 1 " state (where one junction is in the voltage state and $\mathrm{N}-1$ junctions are in the supercurrent state) etc. The bias current at which the array switches from the state " $\mathrm{i}$ " we label $\mathrm{I}_{\mathrm{c}, \mathrm{i}}$.

For comparatively large (i.e., cross-sectional area $>1 \mu \mathrm{m}^{2}$ ) IJJ arrays, the temperature-dependence of $\mathrm{I}_{\mathrm{c}, 0}$ follows the classical Ambegaokar-Baratoff form, being approximately independent of temperature for $\mathrm{T}<\mathrm{T}_{\mathrm{c}} / 2[1,2]$. For sub-micron IJJ arrays, however, $\mathrm{I}_{\mathrm{c}, 0}$ follows an anomalous temperature-dependence, increasing approximately linearly with decreasing temperature even at low temperatures $T<T_{c} / 2$, as a result of thermally activated phase diffusion [2-4]. In this paper, we compare the temperature dependences of the critical currents of the " 0 ", " 1 " and " 2 " states of a sub-micron IJJ array and show that, although $I_{c, 0}(T)$ is approximately linear at low $T, I_{c, 1}(T)$ and $I_{c, 2}(T)$ are temperature-independent at low $\mathrm{T}$.

Our IJJ arrays are fabricated from c-axis-oriented $\mathrm{Tl}_{2} \mathrm{Ba}_{2} \mathrm{CaCu}_{2} \mathrm{O}_{8}$ thin films using focussed ion-beam milling. Current-biased measurements were performed in a liquid-helium dewar. Full experimental details may be found elsewhere [5].

Figure 1 shows the temperature dependences of the critical currents of the " 0 ", " 1 " and " 2 " states for a stack of cross-sectional dimensions $0.6 \mu \mathrm{m} \times 1 \mu \mathrm{m}$ and height $200 \mathrm{~nm}$. As the temperature is reduced, $I_{c, 0}$ continues to increase approximately linearly, consistent with previous measurements by us and others on sub-micron junctions $[2,4]$. This temperature dependence is characteristic of thermally activated phase-diffusion (TAPD) in IJJs. TAPD occurs in hysteretic junctions when the resistance of the $\mathrm{IJJ}$ is sufficiently large that the dissipation seen by the $\mathrm{IJ}$ at the escape frequency is dominated by the shunting impedance, $Z$, of the leads attached to the IJJ. This impedance is typically frequency-dependent and of order $\sim 100 \Omega[6]$.

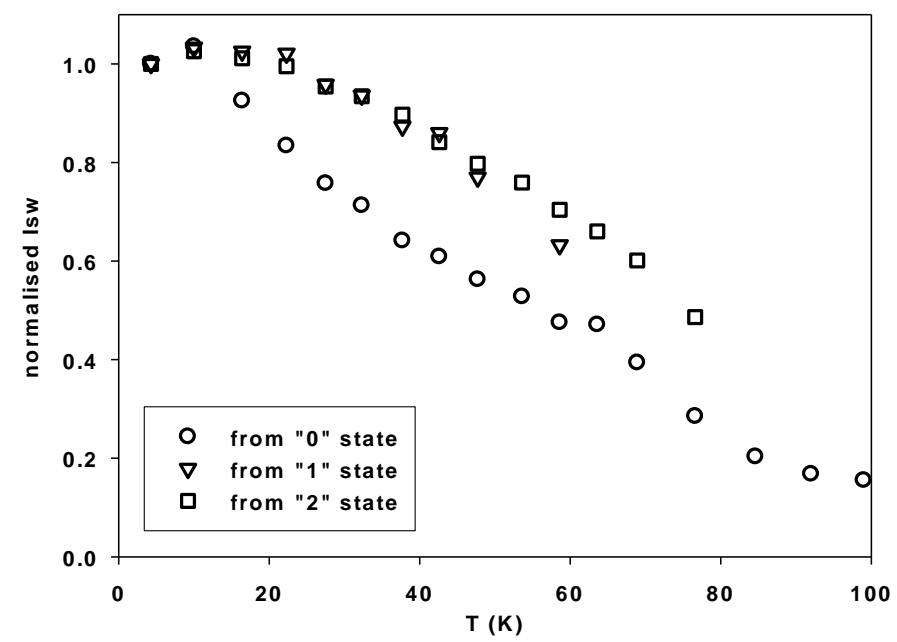

Fig. 1. Variation of switching current with temperature for jumps from states " 0 ", " 1 " and " 2 ". Currents are normalised to their $4.2 \mathrm{~K}$ value.

$I_{c, 1}$ and $I_{c, 2}$ show a different temperature dependence which is constant at low temperature and more reminiscent of the Ambegaokar-Baratoff behaviour of larger IJJs. The question therefore arises: why do we observe these two different temperature dependences in a single $\mathrm{IJJ}$ array containing junctions of the same area and therefore (at least) similar resistance? This behaviour can be accounted for using the equivalent circuit model shown in Fig. 2. In the " 0 " state (Fig. 2(a)) the junctions are shunted by $Z$ leading to phase diffusion. In the "1" state, however, (Fig. 2(b)), there is an additional resistance in the IJJ array - i.e., the resistance of the single junction which is in the voltage state. Measurements of the static current-voltage characteristics show that the junction resistance in state " 1 " is $2 \mathrm{k} \Omega$, which is an order of magnitude larger than $Z$. The 
increase in the impedance seen by the array when it is in the "1" state leads to a corresponding reduction in dissipation at the escape frequency. Hence TAPD is only observed in the " 0 " state and not in higher states.

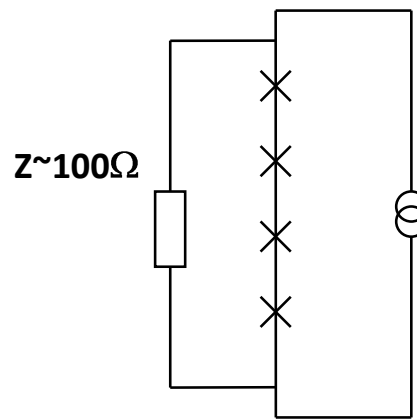

(a)

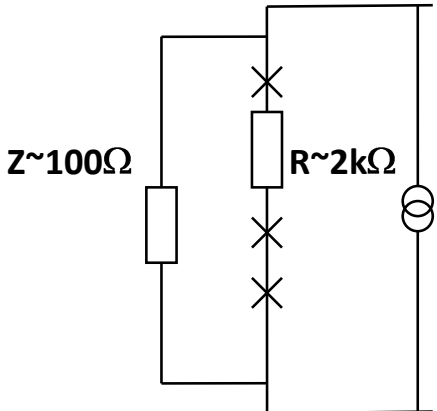

(b)

Fig. 2. Equivalent circuit for (a) state " 0 " and (b) state " 1 ". For clarity, only $4 \mathrm{IJJs}$ are depicted.

This interpretation is supported by measurements of the current-voltage characteristics of an IJJ stack on another sample, as shown in Fig. 3. Here we artificially enhance the apparent phase diffusion voltage by adding an extraneous low frequency periodic pulsed noise source. This causes the voltage in the " 0 " state to oscillate between two values, one of which corresponds to the phasediffusion voltage at the bath temperature while the other corresponds to the phas-diffusion voltage at an effective temperature which is higher as a result of the noise source. The difference between these two voltages is clearly visible in the " 0 " state (i.e. at currents less than $I_{c, 0}$, increasing exponentially with increasing current). The voltage difference is however negligible in the " 1 " state, as expected if TAPD does not occur here.

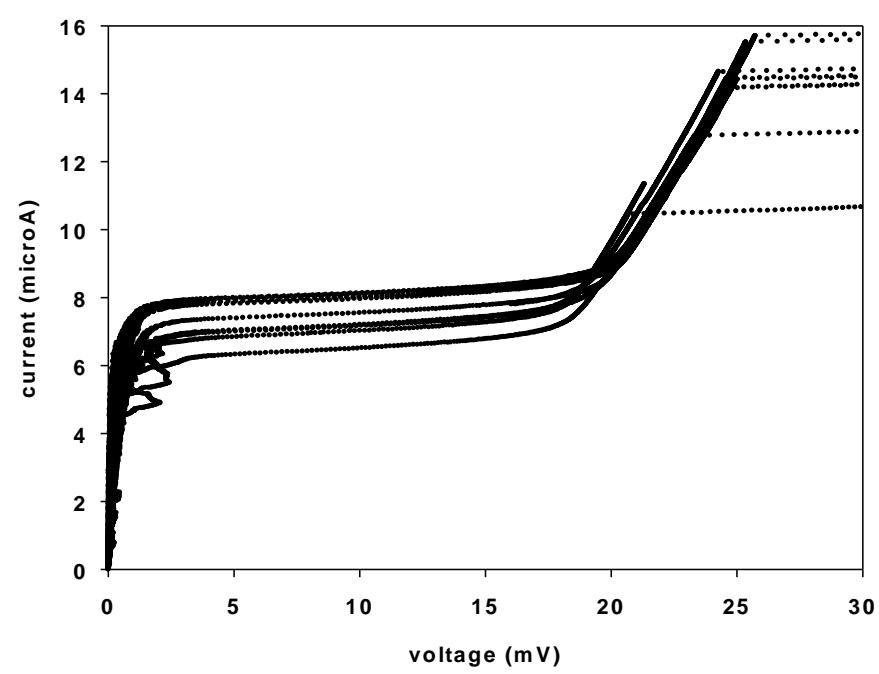

Fig. 3. Time-resolved current-voltage characteristic at $15.9 \mathrm{~K}$ for a stack with cross-sectional area $0.8 \mu \mathrm{m} \times 2 \mu \mathrm{m}$, showing state " 0 " and state " 1 ". Several sweeps at $22 \mathrm{~Hz}$ are shown; successive data points are spaced by $\sim 7 \mu$ s. Near-horizontal portions correspond to jumps between states, which are more rapid than the time response of the measurement system.

We conclude that the temperature dependence of the first and subsequent critical currents is different in sub-micron IJJ arrays because thermally activated phase diffusion is switched off once one junction is in the voltage state. This is due to a corresponding change in the dissipative environment seen by the array. This result suggests that comparisons of macroscopic quantum tunnelling $[7,8]$ out of the two states should reveal illuminating differences.

\section{References}

[1] R. Kleiner, F. Steinmeyer, G. Kunkel, P. Müller, Phys. Rev. Lett. 68 (1992) 2394.

[2] P.A. Warburton et al., J. Appl. Phys. 95 (2004) 4941. 
[3] P.A. Warburton, A.R. Kuzhakhmetov, G. Burnell, M.G. Blamire, H. Schneidewind, Phys. Rev. B 67 (2003) 184513.

[4] A. Franz, Y. Koval, D. Vasyukov, P. Müller, H. Schneidewind, D. A. Ryndyk, J. Keller, C. Helm, Phys. Rev. B 69 (2004) 014506.

[5] P.A. Warburton, et al., IEEE Trans. Appl. Supercond. 13 (2003) 821.

[6] M. Tinkham, Introduction to Superconductivity, 2nd ed.,McGraw-Hill, New York, 1996.

[7] X.Y. Jin, J. Lisenfeld, Y. Koval, A. Lukashenko, A.V. Ustinov, P. Müller, Phys. Rev. Lett. 96 (2006) 177003.

[8] K. Inomata, S. Sato, Koji Nakajima, A. Tanaka, Y. Takano, H.B. Wang, M. Nagao, H. Hatano, S. Kawabata, Phys. Rev. Lett. 95 (2005) 107005. 